Research Article

\title{
Commutativity of MA-Semirings with Involution through Generalized Derivations
}

\author{
Liaqat Ali, ${ }^{1}$ Muhammad Aslam, ${ }^{2}$ Muhammad Imran Qureshi $\left(\mathbb{D},{ }^{3}\right.$ Yaqoub Ahmed Khan, \\ Shafiq Ur Rehman $\left(\mathbb{D},{ }^{5}\right.$ and Ghulam Farid ${ }^{5}$ \\ ${ }^{1}$ Department of Mathematics, Govt. M.A.O College Lahore, Lahore, Pakistan \\ ${ }^{2}$ GC University, Lahore, Pakistan \\ ${ }^{3}$ Department of Mathematics, COMSATS University Islamabad, Vehari Campus, Vehari, Pakistan \\ ${ }^{4}$ Department of Mathematics, Government Islamia College Lahore, Lahore, Pakistan \\ ${ }^{5}$ Department of Mathematics, COMSATS University Islamabad, Attock Campus, Attock, Pakistan
}

Correspondence should be addressed to Shafiq Ur Rehman; shafiq@cuiatk.edu.pk and Ghulam Farid; faridphdsms@hotmail.com

Received 25 August 2020; Accepted 21 November 2020; Published 14 December 2020

Academic Editor: Shaofang Hong

Copyright (C) 2020 Liaqat Ali et al. This is an open access article distributed under the Creative Commons Attribution License, which permits unrestricted use, distribution, and reproduction in any medium, provided the original work is properly cited.

In this paper, we study the generalized derivations of MA-semirings with involution. We discuss some differential identities satisfied by the generalized derivations which force the semirings with involution to be commutative.

\section{Introduction and Preliminaries}

Javed et al. [1] introduced the notion of MA-semiring that is an additive inverse semiring satisfying $A_{2}$ condition of Bandlet and Petrich [2]. The notion of MA-semirings is groundbreaking to use commutators and their related identities in semirings. This enables the algebraists to produce and extend some remarkable results in this area. MAsemiring is a generalized structure of rings and distributive lattices but in spite of semirings we can deal with lie theory in MA-semirings. For ready reference, one can see $[1,3,4]$. The concept of commutators along with derivations and certain additive mappings was further investigated and extended in $[1,3-6]$. Involution is one of the important and fundamental concepts studied in functional analysis and algebra. For instance, $\mathrm{B} *$-algebra due to Rickart [7] and $\mathrm{C}^{*}$-algebra due to Segal [8] are now well-known concepts that are defined with involution. Later on, many algebraists used this idea in groups, rings, and semirings (see [9-22]). Several research papers have been produced for MA-semirings with involution; for reference, one may see $[5,6]$. To discuss the results of rings with involution in MA-semirings with involution would be of great interest for readers and researchers.
In this paragraph, we compose some necessary definitions and preliminary concepts. By a semiring $S$, we mean a semiring with absorbing "0," in which addition is commutative. A semiring $S$ is said to be additive inverse semring if for each $s \in S$ there is a unique $s^{\prime} \in S$ such that $s+s^{\prime}+s=$ $s$ and $s^{\prime}+s+s^{\prime}=s^{\prime}$, where $s^{\prime}$ denotes the pseudoinverse of $s$. An additive inverse semiring $S$ is said to be an MA-semiring if it satisfies $s+s^{\prime} \in Z(S), \forall s \in S$, where $Z(S)$ is the center of $S$. In fact, every ring is MA-semiring, while converse may not be true. The following is one of the examples of MA-semiring which is not a ring. Such examples motivate us to generalize the results of ring theory in MA-semirings.

Example 1 (see [1]). The set $S=\{0,1,2,3,4, \ldots\}$ with addition $\oplus$ and multiplication $\odot$, respectively, defined by $a \oplus b=\sup \{a, b\}$ and $a \odot b=\inf \{a, b\}$ is an MA-semiring. In fact, $S$ is a commutative prime MA-semiring.

Throughout the paper, by $S$ we mean an MA-semiring unless stated otherwise. We say $S$ is prime if $a S b=0$ implies that $a=0$ or $b=0$ and semiprime if $a S a=0$ implies that $a=0 . S$ is 2 -torsion free if, for $s \in S, 2 s=0$ implies $s=0$. An additive mapping $*: S \longrightarrow S$ is involution if, $\forall s, t \in S$, $\left(s^{*}\right)^{*}=s$ and $(s t)^{*}=t^{*} s^{*}$. An element $s \in S$ is Hermitian if 
$s^{*}=s$ and skew Hermitian if $s^{*}=s^{\prime}$. The set of Hermitian elements of $S$ is denoted by $H$ and that of skew Hermitian elements is denoted by $K$.

Example 2 (see [5]). Let $(S,+, \cdot)$ be an MA-semiring. Then the set $S$ with addition "+" and multiplication - defined as $a \cdot b=b . a$ forms an MA-semiring called the opposite MAsemiring of $S$. We usually denote it as $S^{\circ}$. Consider $R=S \times S^{\circ}$ with $(a, b) \oplus(c, d)=(a+c, b+d)$ and $(a, b) \odot(c, d)=$ $(a . c, b \cdot d)=(a c, d b)$. Then $(R, \oplus, \odot)$ forms an MA-semiring. Define $*: R \longrightarrow R$ by $(s, t)^{*}=(t, s)$. Then $*$ defines an involution on $R$. Thus, the triplet $(R, \oplus, \odot)$ forms an MAsemiring with involution. We further see that MA-semiring is *-prime but not prime. Therefore, this example also shows that a prime MA-semiring with involution $*$ is a * -prime MA-semiring but converse is not true in general.

An additive mapping $d: S \longrightarrow S$ is a derivation if $d(s t)=d(s) t+s d(t)$. The concept of generalized derivation was studied for MA-semirings in [6]. An additive mapping $F_{d}: S \longrightarrow S$ is a generalized derivation associated with a derivation $d$ if $F_{d}(s t)=F_{d}(s) t+s d(t)$ (see [23]). The commutator is defined as $[s, t]=s t+t^{\prime} s$. By Jordan product, we mean $s^{\circ} t=s t+t s$ for all $s, t \in S$. A mapping $f: S \longrightarrow S$ is commuting if $[f(s), s]=0, \forall s \in S$. A mapping $f: S \longrightarrow S$ is centralizing if $[[f(s), s], r]=0, \forall s, r \in S$. The following identities are very useful for sequel: for all $s, t, z \in S$,

$$
\begin{aligned}
& \text { (1) }[s, s t]=s[s, t] \\
& \text { (2) }[s t, z]=s[t, z]+[s, z] t \\
& \text { (3) }[s, t z]=[s, t] z+t[s, z] \\
& \text { (4) }[s, t]+[t, s]=t\left(s+s^{\prime}\right)=s\left(t+t^{\prime}\right) \\
& \text { (5) }(s t)^{\prime}=s^{\prime} t=s t^{\prime} \\
& \text { (6) }[s, t]^{\prime}=\left[s, t^{\prime}\right]=\left[s^{\prime}, t\right] \\
& \text { (7) } s \circ(t+z)=s \circ t+s \circ z
\end{aligned}
$$

For more details, one can see $[1,4]$.

In the following, we recall a few results for MA-semirings with involution, which are very useful for proving the main results.

Lemma 1 (see [24]). Let $S$ be a semiprime $M A$-semiring with involution * of second kind. Then $K \cap Z(S) \neq 0$ and therefore $H \cap Z(S) \neq 0$.

Remark 1. Let $S$ be an MA-semiring with involution * of second kind.

(1) For any $k \in K, k^{2} \in H$

(2) For any $h \in H \cap Z(S)$ and $h_{o} \in H, h h_{0} \in H$

Idrissi and Oukhtite [25] proved some results on generalized derivations satisfying certain conditions on rings with involution. The main objective of this paper is to prove the results for MA-semirings with involution.

\section{Main Results}

Lemma 2. Let $S$ be a 2-torsion free prime $M A$-semiring and let $F_{d}$ be a generalized derivation associated with a derivation $d$ of $S$. If $F_{d}$ satisfies

$$
\left[F_{d}[s, t], r\right]=0, \quad \forall s, t, r \in S,
$$

then $S$ is commutative.

Proof. By the hypothesis, for all $s, t, r \in S$, we have $\left[F_{d}(s t)+\right.$ $\left.F_{d}\left(t s^{\prime}\right), r\right]=0$ and therefore

$$
\left[F_{d}(s) t+s d(t)+F_{d}(t) s^{\prime}+t d\left(s^{\prime}\right), r\right]=0 .
$$

If $d=0$, then (2) becomes

$$
\left[F_{d}(s) t+F_{d}(t) s^{\prime}, r\right]=0
$$

In (3), replacing $t$ by $t s$, we get

$$
\left[\left(F_{d}(s) t+F_{d}(t) s^{\prime}\right) s, r\right]=0
$$

From (3), we also have $F_{d}(s) t+F_{d}(t) s^{\prime} \in Z(S)$ and therefore (4) becomes

$$
\left(F_{d}(s) t+F_{d}(t) s^{\prime}\right)[s, r]=0 .
$$

In (5), replacing $r$ by $r w$ and using (5), we obtain

$$
\left(F_{d}(s) t+F_{d}(t) s^{\prime}\right) S[s, w]=0 .
$$

By the primeness of $S$, we get $[s, w]=0$ or $F_{d}(s) t+F_{d}(t) s^{\prime}=0$. If $[s, w]=0$, then $S$ is commutative. Secondly, if

$$
F_{d}(s) t+F_{d}(t) s^{\prime}=0
$$

it also implies

$$
F_{d}(s) t=F_{d}(t) s .
$$

In (7), replacing $t$ by $t r$, we get $F_{d}(s) t r+F_{d}(t) r x^{\prime}=0$ and, using (8), we obtain

$$
F_{d}(t)[s, r]=0
$$

In (9), replacing $s$ by $s w$ and using (9) again, we get $F_{d}(t) S[w, r]=0$. As $S$ is prime and $F_{d} \neq 0,[w, r]=0$. Therefore, $S$ is commutative.

We now consider the case when $d \neq 0$. In (2), replacing $t$ by $t s$ and using (2) again, we have

$$
F_{d}[s, t][s, r]+[[s, t], r] d(s)=0,
$$

which further gives 


$$
F_{d}[s, t][s, r]=[[s, t], r]^{\prime} d(s) .
$$

In (10), replacing $r$ by $r w$, we obtain

$$
\begin{aligned}
& F_{d}[s, t] r[s, w]+F_{d}[s, t][s, r] w+r[[s, t], w] d(s) \\
& \quad+[[s, t], r] w d(s)=0 .
\end{aligned}
$$

From (2), using $F_{d}[s, t] \in Z(S)$ in the last expression, we get

$$
\begin{aligned}
& r\left(F_{d}[s, t][s, w]+[[s, t], w] d(s)\right)+F_{d}[s, t][s, r] w \\
& \quad+[[s, t], r] w d(s)=0,
\end{aligned}
$$

and, using (10), we obtain

$$
F_{d}[s, t][s, r] w+[[s, t], r] w d(s)=0 .
$$

Using (11) into (14), we get $[[s, t], r][w, d(s)]=0$ and so $[[s, t], r] S[w, d(s)]=0$. By the primeness of $S$, we have either $[[s, t], r]=0$ or $[w, \mathrm{~d}(s)]=0$. If $[w, d(s)]=0$, then $[d(w), w]=0, \forall w \in G$, and therefore, by Theorem 2.2 of [26], $S$ is commutative. Secondly, if $[[s, t], r]=0$, then, replacing $t$ by $t s$, we obtain $[s, t][s, r]=0$ and hence $[s, t] S[s, r]=0$. By the primeness of $S$, we conclude that $S$ is commutative.

Lemma 3. Let $S$ be a 2-torsion free prime MA-semiring with involution of second kind. If $S$ satisfies

$$
\left[s \circ t+t^{*} \circ s^{*}, r\right]=0, \quad \forall s, t, r \in S,
$$

then $S$ is commutative.

Proof. By the hypothesis, for all $s, t, r \in S$, we have

$$
\left[s \circ t+t^{*} \circ s^{*}, r\right]=0 \text {. }
$$

In (16), replacing $t$ by $k \in K \cap Z(S)$, we get $\left[s \circ k+k^{*} \circ s^{*}, r\right]=0$, which implies $\left[s \circ k+k^{\prime} \circ s^{*}, r\right]=0$ and therefore $2 k\left[s+s^{*^{\prime}}, r\right]=0$. By the 2-torsion freeness of $S$, we have $k\left[s+s^{*^{\prime}}, r\right]=0$, which further means $k S\left[s+s^{*}, r\right]=0$. In view of Lemma $1, K \cap Z(S) \neq 0$. Therefore, by the primeness of $S$, we obtain $\left[s+s^{*^{\prime}}, r\right]=0$, which is further simplified to be

$$
[s, r]=\left[s^{*}, r\right] .
$$

In (16), replacing $t$ by $h \in H \cap Z(S)$, we get $\left[s \circ h+h^{*} \circ s^{*}, r\right]=0$ and therefore $2 h\left[s+s^{*}, r\right]=0$. By the 2-torsion freeness of $S$, we get $h\left[s+s^{*}, r\right]=0$ and therefore $h S\left[s+s^{*}, r\right]=0$. In the view of Lemma $1, H \cap Z(S) \neq 0$; therefore, by the primeness of $S$, we have $\left[s+s^{*}, r\right]=0$ and hence

$$
[s, r]+\left[s^{*}, r\right]=0,
$$

and, using (17) into (18), we obtain $2[s, r]=0$ which, by the 2 -torsion freeness of $S$, gives $[s, r]=0$. This proves that $S$ is commutative.

Theorem 1. Let $S$ be a 2-torsion free prime MA-semiring with involution * and let $F_{d}$ be a nonzero generalized derivation associated with a derivation d of $S$. If $F_{d}$ satisfies

$$
\left[F_{d}\left[s, s^{*}\right], r\right]=0, \quad \forall s, r \in S,
$$

then $S$ is commutative.

Proof. Firstly, suppose that $d \neq 0$. Linearizing (19) and using (19) again, we get

$$
\left[F_{d}\left[s, t^{*}\right]+F_{d}\left[t, s^{*}\right], r\right]=0 .
$$

In (20), replacing $t$ by $t^{*}$, we get

$$
\left[F_{d}[s, t]+F_{d}\left[t^{*}, s^{*}\right], r\right]=0 .
$$

In (21), replacing $t$ by $t k, k \in K \cap Z(S)$, we get $\left[F_{d}([s, t] k)+F_{d}\left(\left[t^{*}, s^{*}\right] k^{\prime}\right), r\right]=0 . \quad$ As $\quad d=0, \quad k\left[F_{d}[s, t]\right.$ $\left.+F_{d}\left[t^{*}, s^{*}\right]^{\prime}, r\right]=0$, which further implies $k S\left[F_{d}[s, t]\right.$ $\left.+F_{d}\left[t^{*}, s^{*}\right]^{\prime}, r\right]=0$. In view of Lemma 1 , by the primeness of $S$, we get $\left[F_{d}[s, t]+F_{d}\left[t^{*}, s^{*}\right]^{\prime} r\right]=0$ and therefore

$$
\left[F_{d}[s, t], r\right]=\left[F_{d}\left[t^{*}, s^{*}\right], r\right] .
$$

Using (22) into (21) and hence using 2-torsion freeness of $S$, we get $\left[F_{d}[s, t], r\right]=0$. and by Lemma 2 we conclude that $S$ is commutative.

Secondly, suppose that $d \neq 0$.

In (21), replacing $t$ by $y h, h \in H \cap Z(S)$, we obtain $\left[F_{d}([s, t] h)+F_{d}\left(\left[t^{*}, s^{*}\right] h\right), r\right]=0$ and so $\left[F_{d}[s, t] h+[s, t]\right.$ $\left.d(h)+F_{d}\left[t^{*}, s^{*}\right] h+\left[t^{*}, s^{*}\right] d(h), r\right]=0$. Rearranging the terms, we get

$$
\left[F_{d}[s, t]+F_{d}\left[t^{*}, s^{*}\right], r\right] h+\left[\left([s, t]+\left[t^{*}, s^{*}\right]\right) d(h), r\right]=0 .
$$

Using (21) into (23), we get

$$
\left[[s, t]+\left[t^{*}, s^{*}\right] d(h), r\right]=0 .
$$

In (24), replacing $t$ by $t k, k \in K \cap Z(S)$, we obtain $\left[[s, t]+\left[t^{*}, s^{*}\right]^{\prime} d(h), r\right] k=0$ and therefore $[[s, t]+$ $\left.\left[t^{*}, s^{*}\right]^{\prime} d(h), r\right] S k=0$. By the primeness $S$, we have $[[s, t]+$ $\left.\left[t^{*}, s^{*}\right]^{\prime} d(h), r\right]=0$ and therefore

$$
\left[[s, t] d(h), r=\left[t^{*}, s^{*}\right] d(h), r\right] .
$$

Using (23) into (24) and hence using 2-torsion freeness of $S$, we get $[[s, t] d(h), r]=0$, which can be further written as

$$
[[s, t], r] d(h)+[s, t][d(h), r]=0 .
$$

In (26), replacing $t$ by $s t$, we get $[s[s, t], r]$ $d(h)+s[s, t][d(h), r]=0$ and therefore $s[[s, t], r] d(h)$ $+[s, t][s, r] d(h)+s[s, t][d(h), r]=0 . \quad$ Rearranging the terms, $s([[s, t], r] d(h)+[s, t][d(h), r])+[s, t][s, r] d(h)=0$. Using (26) again, we obtain $[s, t][s, r] d(h)=0$. Therefore, we can easily obtain $[s, t][s, r] S d(h)=0$ which, by the primeness, gives that either $S$ is commutative or $d(h)=0$. Suppose that

$$
d(h)=0 .
$$

In the view of Lemma 1 , for any $k \in K \cap Z(S)$, $k^{2} \in H \cap Z(S)$; therefore, replacing $h$ by $k^{2}$ in (27), we get $k S d(k)=0$, and, by the primeness of $S$, we have $d(k)=0$. 
Replacing $t$ by $t k, k \in K \cap Z(S)$ in $(21)$, we get $\left[\left(F_{d}[s, t]+\right.\right.$ $\left.\left.F_{d}\left[t^{*}, s^{*}\right]^{\prime}\right), r\right] k=0$ and therefore $\left[\left(F_{d}[s, t]+\right.\right.$ $\left.\left.F_{d}\left[t^{*}, s^{*}\right]^{\prime}\right), r\right] S k=0$. In the view of Lemma 1, using the primeness of $S$, we have $\left[\left(F_{d}[s, t]+F_{d}\left[t^{*}, s^{*}\right]^{\prime}\right), r\right]=0$, which further implies

$$
\left.\left[F_{d}[s, t], r=F_{d}\left[t^{*}, s^{*}\right]\right), r\right] .
$$

Using (28) into (21) and then using 2-torsion freeness of $S$, we obtain $\left[F_{d}[s, t], r\right]=0$. Employing Lemma 2, we get the required result.

Proposition 1. Let $S$ be 2-torsion free prime MA-semiring with involution of second kind and let $F_{d}$ be a generalized derivation associated with a derivation $d$ of $S$. If $F_{d} \neq I$ and

$$
\left[F_{d}\left[s, s^{*}\right]+\left[s, s^{*}\right]^{\prime}, r\right]=0, \quad \forall s, r \in S,
$$

then $S$ is commutative.

Proof. Suppose that $F_{d} \neq I$. Define a mapping $\psi: S \longrightarrow S$ by

$$
\psi(s)=F_{d}(s)+I^{\prime}(s)=F_{d}(s)+s^{\prime},
$$

where $I^{\prime}(s)=s^{\prime}=$ pseudo inverse of $s$. We now prove that $\psi$ is generalized derivation.

Firstly, for any $s, t \in S$,

$$
\begin{aligned}
\psi(s+t) & =F_{d}(s+t)+(s+t)^{\prime}=F_{d}(s)+F_{d}(t)+s^{\prime}+t^{\prime} \\
& =F_{d}(s)+s^{\prime}+F_{d}(t)+t^{\prime}=\psi(s)+\psi(t) .
\end{aligned}
$$

This shows that $\psi$ is additive. Secondly, for any $s, t \in S$,

$$
\begin{aligned}
\psi(s t)= & F_{d}(s t)+(s t)^{\prime}=F_{d}(s) t+s d(t)+s^{\prime} t \\
= & F_{d}(s) t+s^{\prime} t+s d(t)=\left(F_{d}(s)+s^{\prime}\right) t \\
& +s d(t)=\psi(s) t+s d(t) .
\end{aligned}
$$

This shows that $\psi$ is generalized derivation associated with the derivation $d$. From (29), we can write

$$
\left[\psi\left[s, s^{*}\right], r\right]=0, \quad \forall s, r \in S .
$$

Hence, by Theorem 1 , we conclude that $S$ is commutative.

On the similar lines of Proposition 1, we can obtain the following proposition.

Proposition 2. Let $S$ be 2-torsion free prime MA-semiring with involution of second kind and let $F_{d}$ be a generalized derivation associated with a derivation $d$ of $S$. If $F_{d} \neq I^{\prime}$ and

$$
\left[F_{d}\left[s, s^{*}\right]+\left[s, s^{*}\right], r\right]=0, \quad \forall s, r \in S,
$$

then $S$ is commutative.

Theorem 2. Let $S$ be a 2-torsion free prime MA-semiring with involution $*$. If $F_{d}$ is a nonzero generalized derivation satisfying

$$
\left[F_{d}\left(s \circ s^{*}\right), r\right]=0, \quad \forall s, r \in S,
$$

then $S$ is commutative.

Proof. Linearizing (35) and using (35) again, we get

$$
\left[F_{d}\left(s \circ t^{*}\right)+F_{d}\left(t \circ s^{*}\right), r\right]=0,
$$

And, replacing $t$ by $t^{*}$, we further get

$$
\left[F_{d}(s \circ t)+F_{d}\left(t^{*} \circ s^{*}\right), r\right]=0 .
$$

Suppose that $d=0$. In (37), replacing $t$ by $t k, k \in K \cap Z(S)$, we get $\left[F_{d}\left(\left(s^{\circ} t\right) k\right)+F_{d}\left(\left(t^{*} \mathrm{o} s^{*}\right)^{\prime} k\right), r\right]=0$, which further gives $\left[F_{d}\left(s^{d} t\right)+F_{d}\left(t^{*} o^{*}\right)^{\prime}, r\right] S k=0$. Therefore, in the view of Lemma 1, by the primeness of $S$, we obtain

$$
\left[F_{d}((s \circ t))+F_{d}\left(t^{*} \circ s^{*}\right)^{\prime}, r\right]=0,
$$

and this implies

$$
\left[F_{d}(s \circ t), r=F_{d}\left(t^{*} \circ s^{*}\right), r\right] .
$$

Using (39) into (37) and then using 2-torsion freeness of $S$, we obtain

$$
\left[F_{d}(s \circ t), r\right]=0 .
$$

In (40), replacing $t$ by $h \in H \cap Z(S)$, we obtain $\left[F_{d}(s), r\right] h=0$ and therefore $\left[F_{d}(s), r\right] S h=0$. In view of Lemma 1 , by the primeness, we obtain

$$
\left[F_{d}(s), r\right]=0 .
$$

In (41), replacing $s$ by $s t$ and using (41) again, we find $F_{d}(s)[t, r]=0$ and therefore $F_{d}(s) S[t, r]=0$. As $F_{d} \neq 0$, by the primeness, we have $[t, r]=0$. This proves that $R$ is commutative.

Suppose that $d \neq 0$.

In (37), replacing $t$ by $y h, h \in H \cap Z(S)$, we obtain

$$
\left[F_{d}\left((s \circ t)+\left(t^{*} \circ s^{*}\right) h\right), r\right]=0,
$$

and therefore

$$
\left[F_{d}\left((s \circ t)+\left(t^{*} \circ s^{*}\right), r\right] h+\left[\left((s \circ t)+\left(t^{*} \circ s^{*}\right)\right) d(h), r\right]=0 .\right.
$$

Using (37) again, we obtain

$$
\left[\left((s \circ t)+\left(t^{*} \circ s^{*}\right)\right) d(h), r\right]=0 .
$$

In (44), replacing $t$ by $t k, k \in K \cap Z(S)$, we obtain

$$
\left[\left((s \circ t)+\left(t^{*} \circ s^{*}\right)^{\prime}\right) d(h), r\right] k=0 .
$$

In view of Lemma 1, by the primeness of $S$, we have

$$
\left[\left((s \circ t)+\left(t^{*} \circ s^{*}\right)^{\prime}\right) d(h), r\right]=0,
$$

and therefore 


$$
[(s \circ t) d(h), r]=\left[\left(t^{*} \circ s^{*}\right) d(h), r\right] .
$$
obtain

Using (47) into (44) and the 2-torsion freeness of $S$, we

$$
[(s \circ t) d(h), r]=0 .
$$

In (48), replacing $t$ by $s t$ and using (48) again, we obtain $[s, r](s \circ t) d(h)=0$ and therefore $[s, r] S(s \circ t) d(h)=0$, which further, by the primeness, implies that either $R$ is commutative or

$$
(s \circ t) d(h)=0 .
$$

From (49), we can write

$$
\operatorname{std}(h)=t s^{\prime} d(h) .
$$

In (49), replacing $t$ by $t r$, we get

$$
\operatorname{strd}(h)+\operatorname{srtd}(h)=0 .
$$

Using (50), we get

$$
[s, t] S d(h)=0 .
$$

By the primeness of $S$, we have that either $S$ is commutative or $d(h)=0$. Suppose that $d(h)=0$. Following the same arguments as those in Theorem 1 , we have $d(k)=0$, $\forall k \in K \cap Z(S)$. In (37), replacing $t$ by $t k, k \in K \cap Z(S)$ and using $d(k)=0$, we obtain $\left[F_{d}\left(s^{\circ} t\right)+F_{d}\left(\left(t^{*} \mathrm{o}^{*}\right)^{\prime}\right), r\right]=0$, which further implies

$$
\left[F_{d}(s \circ t), r=F_{d}\left(t^{*} \circ s^{*}\right), r\right] .
$$

Using (53) into (37) and then using 2-torsion freeness of $S$, we get

$$
\left[F_{d}\left(s^{\circ} t\right), r\right]=0 .
$$

In (54), replacing $t$ by $h \in H \cap Z(S)$ and using the fact that $d(h)=0$, we obtain $\left[F_{d}(s), r\right]=0$ and hence, replacing $s$ by $[u, v]$, we find $\left[F_{d}[u, v], r\right]=0$. By Lemma $2, S$ is commutative.

Proposition 3. Let $S$ be 2-torsion free prime MA-semiring with involution of second kind and let $F_{d}$ be a generalized derivation associated with a derivation $d$ of $S$. If $F_{d} \neq I$ and

$$
\left[F_{d}\left(s \circ s^{*}\right)+\left(s \circ s^{*}\right)^{\prime}, r\right]=0, \quad \forall s, r \in S,
$$

then $S$ is commutative.

Proof. Define $\quad \psi: S \longrightarrow S$ by $\psi(s)=F_{d}(s)+I^{\prime}(s)=$ $F_{d}(s)+s^{\prime}$, where $I^{\prime}(s)=s^{\prime}$. Then, following the same lines of the proof of Proposition 1, we find that $\psi$ is a generalized derivation satisfying

$$
\left[\psi\left(s \circ s^{*}\right), r\right]=0 .
$$

Hence, by Theorem 2, S is commutative.

On the similar lines, we can obtain the following result.
Proposition 4. Let $S$ be 2-torsion free prime MA-semiring with involution of second kind and let $F_{d}$ be a generalized derivation associated with a derivation $d$ of $S$. If $F_{d} \neq I^{\prime}$ and

$$
\left[F_{d}\left(s^{\circ} s^{*}\right)+\left(s^{\circ} s^{*}\right), r\right]=0, \quad \forall s, r \in S,
$$

then $S$ is commutative.

Lemma 4. Let $S$ be 2-torsion free prime MA-semiring with involution of second kind. If

$$
\left[\left[s, s^{*}\right], r\right]=0, \quad \forall s, r \in S,
$$

then $S$ is commutative.

Proof. Linearizing (58) and using (58) again, we get

$$
\left[\left[s, t^{*}\right]+\left[t, s^{*}\right], r\right]=0, \quad \forall s, t, r \in S,
$$

and hence, replacing $t$ by $t^{*}$, we further get

$$
\left[[s, t]+\left[t^{*}, s^{*}\right], r\right]=0 \text {. }
$$

In (60), replacing $t$ by $t k, k \in K \cap Z(S)$, we obtain $\left[[s, t]+\left[t^{*}, s^{*}\right]^{\prime}, r\right] k=0$ and therefore $[[s, t]+$ $\left.\left[t^{*}, s^{*}\right]^{\prime}, r\right] S k=0$. In view of Lemma 1 , using the primeness of $S$, we have $\left[[s, t]+\left[t^{*}, s^{*}\right]^{\prime}, r\right]=0$ and hence

$$
[[s, t], r]=\left[t^{*}, s^{*}\right], r .
$$

Using (61) into (60), we obtain $2[[s, t], r]=0$ and since $S$ is 2 -torsion free,

$$
[[s, t], r]=0 .
$$

Replacing $t$ by $s t$ in (62) and using (62) again, we obtain $[s, t][t, r]=0$ and therefore $[s, t] S[t, r]=0$, which, by the primeness, implies $S$ is commutative.

Lemma 5. Let $S$ be 2-torsion free prime MA-semiring with involution of second kind. If

$$
\left[s \circ s^{*}, r\right]=0, \quad \forall s, r \in S,
$$

then $S$ is commutative.

Proof. Linearizing (63) and using (63) again, we obtain $\left[s^{\circ} t^{*}+t^{\circ} s^{*}, r\right]=0$ and hence, replacing $t$ by $t^{*}$, we obtain

$$
\left[s \circ t+t^{*} \circ s^{*}, r\right]=0 \text {. }
$$

In (64), replacing $t$ by $t k, k \in K \cap Z(S)$, we obtain $[s \circ t+$ $\left.\left(t^{*} \circ s^{*}\right)^{\prime}, r\right] k=0$ and therefore $\left[s \circ t+\left(t^{*} \circ s^{*}\right)^{\prime}, r\right] S k=0$. In view of Lemma 1 , we get $\left[s \circ t+\left(t^{*} \circ s^{*}\right)^{\prime}, r\right]=0$ and hence

$$
[s \circ t, r]=\left[t^{*} \circ s^{*}, r\right] \text {. }
$$

As $S$ is 2-torsion free, using (65) into (64), we get

$$
[s \circ t, r]=0 .
$$

In (66), replacing $t$ by $h \in H \cap Z(S)$, we get $[s, r] S h=0$ and, employing Lemma 1 , we conclude that $S$ is commutative. 
Theorem 3. Let $S$ be 2-torsion free prime MA-semiring with involution of second kind and let $F_{d}$ be a generalized derivation associated with a derivation d of $S$. If $F_{d}$ satisfies

$$
\left[F_{d}\left(s \circ s^{*}\right)+\left[s, s^{*}\right], r\right]=0, \quad \forall s, r \in S,
$$

then $S$ is commutative.

Proof. When $F_{d}=0$, (67) becomes $\left[\left[s, s^{*}\right], r\right]=0, \forall s, r \in S$. Therefore, employing Lemma 4 , we conclude that $S$ is commutative. When $F_{d} \neq 0$, firstly suppose that $d=0$. Linearizing (67) and using it again, we obtain

$$
\left[F_{d}\left(s \circ t^{*}\right)+F_{d}\left(t \circ s^{*}\right)+\left[s, t^{*}\right]+\left[t, s^{*}\right], r\right]=0,
$$

And, replacing $t$ by $t^{*}$, we obtain

$$
\left[F_{d}(s \circ t)+F_{d}\left(t^{*} \circ s^{*}\right)+[s, t]+\left[t^{*}, s^{*}\right], r\right]=0 .
$$

In (69), replacing $t$ by $t k, k \in K \cap Z(S)$ and using the assumption $d=0$, we obtain

$$
\left[F_{d}(s \circ t)+F_{d}\left(\left(t^{*} \circ s^{*}\right)^{\prime}\right)+[s, t]+\left[t^{*}, s^{*}\right]^{\prime}, r\right] S k=0 .
$$

By the primeness of $S$, we get

$$
\left[F_{d}(s \circ t)+F_{d}\left(\left(t^{*} \circ s^{*}\right)^{\prime}\right)+[s, t]+\left[t^{*}, s^{*}\right]^{\prime}, r\right]=0,
$$

and hence

$$
\left[F_{d}(s \circ t)+[s, t], r\right]=\left[F_{d}\left(\left(t^{*} \circ s^{*}\right)\right)+\left[t^{*}, s^{*}\right], r\right] .
$$

As $S$ is 2-torsion free, using (72) into (69), we get

$$
\left[F_{d}(s \circ t)+[s, t], r\right]=0 .
$$

From (73), we can write

$$
\left[F_{d}(s \circ t), r\right]=[[s, t], r]^{\prime}=[[t, s], r] .
$$

Interchanging $s$ and $t$ in (74), we obtain $\left[F_{d}(t \circ s), r\right]$ $=[[s, t], r]$. But $t \circ s=s \circ t$; therefore

$$
\left[F_{d}(s \circ t), r\right]=[[s, t], r] \text {. }
$$

Using (75) into (74), we get $2[[s, t], r]=0$ and, by the 2 torsion freeness of $S$, we get

$$
[[s, t], r]=0 .
$$

In (76), replacing $t$ by $s t$ and using (76) again, we obtain $[s, t][s, r]=0$ and therefore $[s, t] R[s, r]=0$. By the primeness of $S$, we conclude that $S$ is commutative.

Next, suppose that $d \neq 0$.

In (69), replacing $t$ by $t h, h \in H \cap Z(S)$ and using (69) again, we obtain

$$
\left[[s, t]+\left[t^{*}, s^{*}\right] d(h), r\right]=0 .
$$

In (77), replacing $t$ by $t k, k \in K \cap Z(S)$, we obtain $\left[[s, t]+\left[t^{*}, s^{*}\right]^{\prime} d(h), r\right] w=0$ and therefore $[[s, t]+$ $\left.\left[t^{*}, s^{*}\right]^{\prime} d(h), r\right] S w=0$. In view of Lemma 1 , by the primeness of $S$, we obtain $\left[\left([s, t]+\left[t^{*}, s^{*}\right]^{\prime}\right) d(h), r\right]=0$, which further gives

$$
[[s, t] d(h), r]=\left[\left[t^{*}, s^{*}\right] d(h), r\right] .
$$

As $S$ is 2-torsion free, using (78) into (77), we get

$$
[[s, t] d(h), r]=0 .
$$

In (79), replacing $t$ by $t s$ and using (79), we obtain $[s, t] d(h)[s, r]=0$ and therefore $[s, t] d(h) S[s, r]=0$. By the primeness of $S$, we get $[s, t] d(h)=0$ and therefore as above we conclude that either $S$ is commutative or $d(h)=0$ and this further implies $d(k)=0, \forall k \in K \cap Z(S)$. In (69), replacing $t$ by $t h, h \in H \cap Z(S)$ and using the fact that $d(h)=0$, we obtain $\left[F_{d}(s \circ t)+F_{d}\left(t^{*} \circ s^{*}\right), r\right] h=0$, which further implies $\left[F_{d}(s \circ t)+F_{d}\left(t^{*} \circ s^{*}\right), r\right] S h=0$. In view of Lemma 1, we have

$$
\left[F_{d}(s \circ t)+F_{d}\left(t^{*} \circ s^{*}\right), r\right]=0 .
$$

In (69), replacing $t$ by $t k, k \in K \cap Z(S)$ and using the fact that $d(k)=0$, we obtain $\left[F_{d}(s \circ t)+\left(F_{d}\left(t^{*} \circ s^{*}\right)\right)^{\prime}, r\right] k=0$, which further implies $\left[F_{d}(s \circ t)+\left(F_{d}\left(t^{*} \circ s^{*}\right)\right)^{\prime}, r\right] S k=0$. In view of Lemma 1 , we obtain $\left[F_{d}\left(s^{\circ} t\right)+\left(F_{d}\left(t^{* \circ} s^{*}\right)\right)^{\prime}, r\right]=0$ and hence

$$
\left[F_{d}(s \circ t), r\right]+\left[F_{d}\left(t^{*} \circ s^{*}\right), r\right] .
$$

Using (81) into (80) and then by the 2-torsion freeness of $S$, we obtain $\left[F_{d}[s \circ t], r\right]=0$. Following the same steps as Theorem 2 (equation (40)), we conclude that $S$ is commutative.

On the similar lines of Theorem 3, we can establish the following result.

Theorem 4. Let $S$ be 2-torsion free prime MA-semiring with involution of second kind and let $F_{d}$ be a generalized derivation associated with a derivation $d$ of $S$. If $F_{d}$ satisfies

$$
\left[F_{d}\left(s \circ s^{*}\right)+\left[s, s^{*}\right]^{\prime}, r\right]=0, \quad \forall s, r \in S,
$$

then $S$ is commutative.

Theorem 5. Let $S$ be 2-torsion free prime MA-semiring with involution of second kind and let $F_{d}$ be a generalized derivation associated with a derivation $d$ of $S$. If $F_{d}$ satisfies

$$
\left[F_{d}\left[s, s^{*}\right]+\left(s \circ s^{*}\right), r\right]=0, \quad \forall s, r \in S,
$$

then $S$ is commutative.

Proof. Suppose that $F_{d}=0$. Then (83) becomes $\left[\left(s^{\circ} s^{*}\right), r\right]=0, \forall s, r \in S$. By Lemma $5, S$ is commutative.

Now suppose that $F_{d} \neq 0$. Consider the case when $d=0$. Linearizing (83) and using (83) again, we get

$$
\left[F_{d}\left[s, t^{*}\right]+F_{d}\left[t, s^{*}\right]+\left(s \circ t^{*}\right)+\left(t \circ s^{*}\right), r\right]=0 .
$$

In (84), replacing $t$ by $t^{*}$, we obtain

$$
\left[F_{d}[s, t]+F_{d}\left[t^{*}, s^{*}\right]+(s \circ t)+\left(t^{*} \circ s^{*}\right), r\right]=0 .
$$

In (85), replacing $t$ by $t k, k \in K \cap Z(S)$ and using the assumption $d=0$, we get 


$$
\left[F_{d}[s, t] k+F_{d}\left[t^{*}, s^{*}\right] k^{\prime}+(s \circ t) k+\left(t^{*} \circ s^{*}\right) k^{\prime}, r\right]=0,
$$

and therefore, by rearrangement, we have

$$
\left[F_{d}[s, t]+(s \circ t)+\left(F_{d}\left[t^{*}, s^{*}\right]+\left(t^{*} \circ s^{*}\right)\right)^{\prime}, r\right] k=0,
$$

and hence

$$
\left[F_{d}[s, t]+(s \circ t)+\left(F_{d}\left[t^{*}, s^{*}\right]+\left(t^{*} \circ s^{*}\right)\right)^{\prime}, r\right] S k=0 .
$$

In view of Lemma 1 , by the primeness of $S$, we obtain

$$
\left[F_{d}[s, t]+(s \circ t)+\left(F_{d}\left[t^{*}, s^{*}\right]+\left(t^{*} \circ s^{*}\right)\right)^{\prime}, r\right]=0,
$$

which further gives

$$
\left[F_{d}[s, t]+(s \circ t), r\right]=\left[F_{d}\left[t^{*}, s^{*}\right]+\left(t^{*} \circ s^{*}\right), r\right],
$$

Using (90) into (85) and the 2-torsion freeness of $S$, we obtain

$$
\left[F_{d}[s, t]+(s \circ t), r\right]=0 .
$$

In (85), interchanging $s$ and $t$, we get $\left[F_{d}[t, s]+\right.$ $t \circ s, r]=0$. But since $s^{\circ} t=t^{\circ} s,\left[F_{d}[t, s]+s \circ t, r\right]=0$, which implies $\left[\left(F_{d}[s, t]\right)^{\prime}+s^{\circ} t, r\right]=0$. Hence,

$$
\left[F_{d}[s, t], r\right]=[s \circ t, r] .
$$

As $S$ is 2-torsion free, using (92) into (91), we find

$$
[s \circ t, r]=0 .
$$

In (93), replacing $t$ by $h \in H \cap Z(S)$ and then using 2torsion freeness of $S$, we get $h[s, r]=0$ and therefore $h S[s, r]=0$. In view of Lemma 1 , by the primeness of $S$, we get $[s, r]=0$. This shows that $R$ is commutative.

Next, suppose that $d \neq 0$.

In (85), replacing $t$ by $y h, h \in H \cap Z(S)$, we get

$$
\left[F_{d}[s, t] h+[s, t] d(h)+F_{d}\left[t^{*}, s^{*}\right] h+\left[t^{*}, s^{*}\right] d(h)+(s \circ t) h+\left(t^{*} \circ s^{*}\right) h, r\right]=0 .
$$

Rearranging terms, we can write

$$
\left[F_{d}[s, t]+F_{d}\left[t^{*}, s^{*}\right]+\left(s^{\circ} t\right)+\left(t^{*} \circ s^{*}\right), r\right] h+\left[\left([s, t]+\left[t^{*}, s^{*}\right]\right) d(h), r\right]=0 .
$$

$$
\left[F_{d}[s, t]+(s \circ t), r\right]=\left[F_{d}\left[t^{*}, s^{*}\right]+\left(t^{*} \circ s^{*}\right), r\right] \text {. }
$$

Using (85) again, we get

$$
\left[\left([s, t]+\left[t^{*}, s^{*}\right]\right) d(h), r\right]=0 .
$$

In (96), replacing $t$ by $t k, k \in K \cap Z(S)$, we obtain $\left[\left([s, t]+\left[t^{*}, s^{*}\right]^{\prime}\right) d(h), r\right] k=0$, which further gives $\left[\left([s, t]+\left[t^{*}, s^{*}\right]^{\prime}\right) d(h), r\right] S w=0$. As $S$ is prime, employing Lemma 1 , we get $\left[[s, t] d(h)+\left[t^{*}, s^{*}\right]^{\prime} d(h), r\right]=0$ and hence

$$
\left[[s, t] d(h), r=\left[t^{*}, s^{*}\right] d(h), r\right] .
$$

Using (97) into (96) and hence using 2-torsion freeness of $S$, we obtain

$$
[[s, t] d(h), r]=0 .
$$

Equation (98) is the same as (79) of Theorem 3; therefore, following the same steps, we conclude that either $S$ is commutative or $d(h)=0$. If $d(h)=0$, then $d(k)=0, \forall k \in K \cap Z(S)$.

Rearranging the terms of (85), we get

$$
\left[F_{d}[s, t]+(s \circ t)+F_{d}\left[t^{*}, s^{*}\right]+\left(t^{*} \circ s^{*}\right), r\right]=0 .
$$

In (99), replacing $t$ by $t k, k \in K \cap Z(S)$ and using the fact that $d(k)=0$, we get

$$
\left[F_{d}[s, t]+(s \circ t)+F_{d}\left[t^{*}, s^{*}\right]^{\prime}+\left(t^{*} \circ s^{*}\right)^{\prime}, r\right] k=0,
$$

which further implies
Using (101) into (99) and then by the 2-torsion freeness of $S$, we have

$$
\left[F_{d}[s, t]+(s \circ t), r\right]=0
$$

Interchanging $s$ and $t$ in (102), we obtain $\left[F_{d}[t, s]+(t \circ s), r\right]=0$. As $t \circ s=s \circ t$, the last equation becomes $\left[\left(F_{d}[s, t]\right)^{\prime}+s \circ t, r\right]=0$. This implies that

$$
\left[F_{d}[s, t]=[s \circ t, r]\right. \text {. }
$$

Using (103) into (102), we get $2[s \circ t, r]=0$ and by the 2 torsion freeness of $S$, we have

$$
[s \circ t, r]=0 \text {. }
$$

In (104), replacing $t$ by $h \in H \cap Z(S)$, we obtain $2 h[s, r]=0$ and by the 2-torsion freeness of $S$, it is implied that $h[s, r]=0$ and therefore $h S[s, r]=0$. In view of Lemma 1 , by the primeness of $S$, we conclude that $S$ is commutative.

In view of the above results, we can easily conclude the following results.

Theorem 6. Let $S$ be 2-torsion free prime MA-semiring with involution of second kind and let $F_{d}$ be a generalized derivation associated with a derivation $d$ of $S$. Then the following statements are equivalent: 

(i) $[S, S]=0$
(ii) $\left[F_{d}\left(s \circ s^{*}\right)+\left[s, s^{*}\right], r\right]=0, \forall s, r \in S$
(iii) $\left[F_{d}\left[s, s^{*}\right]+\left(s \circ s^{*}\right), r\right]=0, \forall s, r \in S$

Theorem 7. Let $S$ be 2-torsion free prime MA-semiring with involution of second kind and let $F_{d}$ be a generalized derivation associated with a derivation $d$ of $S$. Then the following statements are equivalent:
(i) $[S, S]=0$
(ii) $\left[F_{d}\left(s^{\circ} s^{*}\right)+\left[s, s^{*}\right]^{\prime}, r\right]=0, \forall s, r \in S$
(iii) $\left[F_{d}\left[s, s^{*}\right]+\left(s \circ s^{*}\right)^{\prime}, r\right]=0, \forall s, r \in S$

\section{Concluding Remarks}

Commutativity is a very important aspect of mathematics and is discussed in almost all of its branches. This article presents some results on generalized derivations of MAsemirings with involution of second kind. This research is useful for researchers who want to induce commutativity in semirings with additive mappings and opens the door for further research in this area. Other differential identities and different mappings can be studied to induce commutativity in semirings.

\section{Data Availability}

No data were used to support for this research.

\section{Conflicts of Interest}

The authors declare that they have no conflicts of interest.

\section{Authors' Contributions}

All authors contributed equally.

\section{References}

[1] M. A. Javed, M. Aslam, and M. Hussain, "On condition $\left(A_{2}\right)$ of bandlet and petrich for inverse semiqrings," International Mathematical Forum, vol. 7, no. 59, pp. 2903-2914, 2012.

[2] H. J. Bandlet and M. Petrich, "Subdirect products of rings and distrbutive lattics," Proceedings of the Edinburgh Mathematical Society, vol. 25, pp. 135-171, 1982.

[3] M. A. Javed and M. Aslam, "Commuting mappings of semiprime MA-semirings," World Applied Sciences Journal (WASJ), vol. 30, no. 1, 2014.

[4] M. Sara, M. Aslam, and M. A. Javed, "On centralizer of semiprime inverse semiring," General Algebra and Applications, vol. 36, pp. 71-84, 2016.

[5] L. Ali, M. Aslam, and Y. A. Khan, "Some commutativity conditions on *-prime semirings," JP Journal of Algebra, Number Theory and Applications, vol. 46, no. 2, pp. 109-121, 2020.

[6] L. Ali, M. Aslam, Y. A. Khan, and G. Farid, "On generalized derivations of semirings with involution," Journal of mechanics of continua and mathematical sciences, vol. 15, no. 04, pp. 138-152, 2020.

[7] C. E. Rickart, "Banach algebras with an adjoint operation," The Annals of Mathematics, vol. 47, no. 3, pp. 528-550, 1946.
[8] I. E. Segal, "Irreducible representations of operator algebras," Bulletin of the American Mathematical Society, vol. 53, no. 2, pp. 73-89, 1947.

[9] R. Awtar, "Lie and Jordan structure in prime rings with derivations," Proceedings of the American Mathematical Society, vol. 41, no. 1, p. 67, 1973.

[10] K. I. Beidar and W. S. Martindale, "On functional identities in prime rings with involution," Journal of Algebra, vol. 203, no. 2, pp. 491-532, 1998.

[11] H. E. Bell, "Martindale, centralizing mappings of semiprime rings centralizing mappings of semiprime rings," Canadian Mathematical Bulletin, vol. 30, no. 1, pp. 92-101, 1987.

[12] J. Martindale, I. N. Herstein, and J. W. Kerr, "Lie ideals ana derivations of prime rings," Journal of Algebra, vol. 71, pp. 259-267, 1981.

[13] M. N. Daif, "Commutativity results for semiprime rings with derivations," International Journal of Mathematics and Mathematical Sciences, vol. 21, no. 3, pp. 471-474, 1998.

[14] I. N. Herstein, Rings with Involution, University of Chicago Press, Chicago, London, UK, 1976.

[15] B. E. Johnson, "Continuity of derivations on commutative algebras," American Journal of Mathematics, vol. 91, no. 1, pp. 1-10, 1969.

[16] D. A. Jordan, "On the ideals of a lie algebra of derivations," Journal of the London Mathematical Society, vol. 2, no. 33, pp. 33-39, 1986.

[17] C. Lanski, "Commutation with skew elements in rings with involution," Pacific Journal of Mathematics, vol. 83, no. 2, pp. 393-399, 1979.

[18] T. K Lee, "On derivations of prime rings with involution," Chinese Journal of Mathematics, vol. 20, no. 02, pp. 191-203, 1992.

[19] L. Oukhtite, "On Jordan ideals and derivations in rings with involution," Commentationes Mathematicae Universitatis Carolinae, vol. 51, no. 03, pp. 389-395, 2010.

[20] L. Oukhtite and A. Mamouni, "Generalized derivations centralizing on jordanideals of rings with involution," Turkish Journal of Mathematics, vol. 38, pp. 225-232, 2014.

[21] E. C. Posner, "Derivations in prime rings," Proceedings of the American Mathematical Society, vol. 8, no. 6, p. 1093, 1957.

[22] S. M. A. Zaidi, M. Ashraf, and S. Ali, "On jordan ideals and left $(\theta, \theta)$-derivations in prime rings," International Journal of Mathematics and Mathematical Sciences, vol. 2004, no. 37, 8 pages, Article ID 296847, 2004.

[23] M. Bresar, "On the distance of the composition of two derivations to the generalized derivations," Glasgow Mathematical Journal, vol. 33, no. 1, pp. 89-93, 1991.

[24] L. Ali, M. Aslam, and Y. A. Khan, "Some results on commutativity of MA-semirings," Indian Journal of Science and Technology, vol. 13, no. 31, pp. 3198-3203, 2020.

[25] A. Idrisi and L. Oukhtite, "Some commutativity theorems for rings with involution involving generalized derivations," Asian-European Journal of Mathematics, vol. 12, no. 01, pp. 1950001-1950012, 2017.

[26] L. Ali, Y. A. Khan, and M. Aslam, "On posner's second theorem for semirings with involution," Journal of Discrete Mathematical Sciences and Cryptography, vol. 23, no. 6, pp. 1195-1202, 2020. 